\title{
Bioactivity Assessment of Poly( $\varepsilon$-caprolactone)/Hydroxyapatite Electrospun Fibers for Bone Tissue Engineering Application
}

\author{
Mohd Izzat Hassan, Naznin Sultana, and Salehhuddin Hamdan \\ Faculty of Biosciences and Medical Engineering, Universiti Teknologi Malaysia (UTM), Skudai, 81310 Johor Bahru, Johor, Malaysia \\ Correspondence should be addressed to Naznin Sultana; naznin@biomedical.utm.my
}

Received 9 May 2014; Accepted 26 June 2014; Published 9 July 2014

Academic Editor: Hao Bai

Copyright ( 2014 Mohd Izzat Hassan et al. This is an open access article distributed under the Creative Commons Attribution License, which permits unrestricted use, distribution, and reproduction in any medium, provided the original work is properly cited.

\begin{abstract}
Electrospinning is useful for fabricating nanofibrous structure with different composition and morphologies. It offers great advantages through its geometrical structure and biomimetic property, which can provide a suitable environmental site for cell growth. The fiber diameter is entangled by the concentration of PCL with some adjustment of parameters during electrospinning process. PCL with lower concentration had bead structure while higher concentration had smooth fiber. The incorporation of nanoparticle hydroxyapatite (nHA) into poly( $\varepsilon$-caprolactone) fiber was studied. The fiber diameter of PCL was increased with the addition of nHA. Composition of fiber at lower concentrations of PCL and nHA into the polymer produced fiber with a homogenous distribution of nHA in PCL fiber with less agglomeration. The immersion of PCL/nHA fiber in simulated body fluid (SBF) had bonelike apatite layer on its surface while PCL showed no results. PCL/nHA showed high water uptake and had improved wettability compared to PCL alone, suggesting that PCL/nHA fibers were more hydrophilic than PCL fiber.
\end{abstract}

\section{Introduction}

Bone is a nanostructured biomaterial with unique biological and mechanical properties. It consists of inorganic hydroxyapatite crystal, organic type I collagen fibers, and other proteins [1]. However, at some time bone may damage and lose its function because of disease or accident. Thus, there is a clinical need for the replacement of the damaged bone. Current trend in clinical practice to overcome the problem is tissue engineering. The purpose of tissue engineering is to regenerate and restore damaged tissue with the combinations of biomaterials, cells, and bioactive agents [2]. In tissue engineering, commonly biodegradable and biocompatible scaffold is used to replace the defect providing a platform for cell function, adhesion, and transplantation.

Nanofibrous scaffold offers great advantages such as the large surface area to volume ratio, with pore structure characteristic, and it can mimic the natural extracellular matrix which is beneficial for cell growth. Among the various techniques of scaffold fabrication, electrospinning can be described as simple and most reliable for producing long and continuous fibers [3-5]. A wide range of polymers can be fabricated using this technique. In addition, the diameters of the fibers can be controlled from a few micrometers to a few nanometers, depending on the optimization during the electrospinning process and parameter such as solvent used [6].

The scaffold may come from natural and synthetic materials. Synthetic polymer poly( $\varepsilon$-caprolactone) (PCL) has been widely used as biomedical materials such as suture material and drug delivery device. It is semicrystalline and bioresorbable aliphatic polyester. It has been intensively explored as tissue engineering scaffold because of its slow biodegradable property $(2-4$ years) $[7,8]$. Due to the low melting temperature at $55-60^{\circ} \mathrm{C}$, the PCL can be easily molded into the desired scaffold design from different fabrication techniques. However, PCL is hydrophobic in nature which results in lack of wettability and poor cell attachment compared to hydrophilic material [9]. One way to modify the surface properties of hydrophobic PCL is by blending with bioresorbable ceramics, such as calcium phosphates, hydroxyapatite (HA), and tricalcium phosphate (TCP). HA exists as a major composition of materials in human bone. The presence of HA in the polymer/composite is advantageous for osteoblast proliferation [10-12]. 
In this study, nanoparticle hydroxyapatite (nHA) incorporation into PCL fiber was investigated through the dispersibility of nHA within the fiber. The surface of the fiber was modified by immersion in a simulated body fluid (SBF) to mimic the mineral of native bone. PCL is a synthetic polymer. So, it may be extremely difficult to induce mineralization because PCL had very few ionic molecular groups compared to natural polymers such as chitosan and collagen. The existence of HA within PCL polymer fibers could improve the surface mineralization process.

\section{Materials and Methods}

2.1. Materials. PCL with molecular weight 70,000 to 90,000 and acetone (99.8\%) were purchased from Sigma-Aldrich. Nanoparticle HA was synthesized by nanoemulsion technique produced in-house from the previous study $[13,14]$.

\subsection{Methods}

2.2.1. Preparation of the Solution. PCL was dissolved in acetone solvent at a concentration of $7.5 \%(\mathrm{w} / \mathrm{v})$ and $12.5 \%(\mathrm{w} / \mathrm{v})$ at $40^{\circ} \mathrm{C}$. HA powder was suspended into the solution at a concentration of $10 \%(\mathrm{w} / \mathrm{w})$ and $20 \%(\mathrm{w} / \mathrm{w})$, respectively. The mixed solution was stirred at room temperature for $24 \mathrm{~h}$ and homogenized using homogenizer (IKA T25, IKA works, Germany).

2.2.2. Electrospinning Fibrous Scaffold. The prepared solution was transferred into a $5 \mathrm{~mL}$ syringe, with 18 and $22 \mathrm{G}$ blunt-end needle. The electrospinning was conducted using electrospinning unit (NaBond, China). The distance between the needle tip and aluminium collector was adjusted at $10 \mathrm{~cm}$. High voltage of $15-22 \mathrm{kV}$ was applied to the needle. The solution was ejected at a feeding rate of $3 \mathrm{~mL} / \mathrm{h}$ using infusion pump (Veryark TCV-IV, China). The resulting fiber was dried overnight to remove any solvent left on its surface.

2.2.3. Bioactivity Test. The bioactivity test was done by immersing the fibers in SBF. The SBF was prepared in accordance with Kokubo et al. [15] with ion concentration (in mM: $\mathrm{Na}^{+} 142.0, \mathrm{~K}^{+} 5.0, \mathrm{Mg}^{2+} 1.5, \mathrm{Ca}^{2+} 2.5, \mathrm{Cl}^{-} 147.8, \mathrm{HCO}_{3}^{-}$ $\left.4.2, \mathrm{HPO}_{4}{ }^{2-} 1.0, \mathrm{SO}_{4}{ }^{2-} 0.5\right)$ almost similar to human blood plasma with initial $\mathrm{pH} 7.4$ at $37^{\circ} \mathrm{C}$ in a water bath (Memmert, Schwabach, Germany). The fiber was cut into $(1 \times 1) \mathrm{cm}^{2}$, left in SBF, and was extracted at 3, 7, and 14 days.

2.2.4. Microstructure Characterization. The morphology of the fibers was observed under SEM (Hitachi TM 3000, Japan) at an accelerating voltage of $15 \mathrm{kV}$ and FESEM (Hitachi SU 8020, Japan). The fibers immersed in SBF analyzed by FESEM were gold-coated prior to analysis. The diameter of single fiber was measured at random location using image analysis software (Image J, NIH, USA). Energy dispersive Xray (EDX) was used to confirm the presence of HA in the PCL polymer.
2.2.5. Contact Angle. Water contact angle was measured by dropping deionized water $(3 \mu \mathrm{L})$ onto the surface using 28 gauge blunt tip needle on different areas on the surfaces to determine the wettability of the fibers using a contact angle instrument (VCA-Optima, AST Inc., USA). Water was dropped on 5/6 places on each sample surface and the average and standard deviation was calculated.

2.2.6. Water Uptake. Water uptake of the fiber was measured to see how much water can be absorbed, which mainly depends on the hydrophilicity of the fiber. The fiber before immersion in SBF was cut into $(2 \times 1) \mathrm{cm}^{2}$ and immersed in deionized water. The fiber was removed at the interval of $1 \mathrm{~min}$, wiped gently with clean tissue paper, and weighed. The process was repeated for $10 \mathrm{~min}$. The water uptake of the fiber after immersion in SBF was measured similarly at different intervals of 3,7 , and 14 days. The water uptake was calculated using the following equation [16]:

$$
\% \text { Water uptake }=100 \times \frac{W_{\text {wet }}-W_{o}}{W_{o}},
$$

where $W_{\text {wet }}$ is the weight of the wet fiber and $W_{o}$ is the starting dry weight of the fiber.

\section{Results and Discussion}

3.1. Morphology of Electrospun Fibrous Scaffold. Figure 1 shows the morphologies of fibrous scaffolds produced from 7.5\% (w/v) PCL, 12.5\% (w/v) PCL, 10\% nHA incorporated $7.5 \%(\mathrm{w} / \mathrm{v})$ and $12.5 \%(\mathrm{w} / \mathrm{v})$ PCL, and $20 \% \mathrm{nHA}$ incorporated 7.5\% (w/v) and 12.5\% (w/v) PCL (Figures 1(a)-1(f)). Electrospun nanofibrous PCL at lower concentration had the disadvantage. There were many unwanted beads with spindlelike structure which was observed (Figure 1(a)). Meanwhile, smooth fiber was obtained at higher concentration (Figure 1(b)). However, there was an increased size of fiber from nanometer into micrometer in range. Incorporation of nHA into PCL fibers was also examined. The addition of $10 \%(\mathrm{w} / \mathrm{w})$ nHA into $7.5 \%(\mathrm{w} / \mathrm{v})$ PCL and $12.5 \%(\mathrm{w} / \mathrm{v}) \mathrm{PCL}$ was found to have smooth fiber structure (Figures $1(\mathrm{c})$ and 1(e)). Increased fiber diameter was also observed. The nHA was well-dispersed into the fiber and only small agglomeration was found. In a recent study, it was described that the agglomeration of nanoparticles resulted in large size and low mechanical properties of fiber [17]. This was related to the increased content of loading nanoparticles. In our study, no apparent change in the size of the fiber was observed. It proves the successful incorporation of nHA nanoparticles homogeneously in the fibers. On the other hand, the addition of $20 \%(\mathrm{w} / \mathrm{w})$ nHA into $12.5 \%(\mathrm{w} / \mathrm{w})$ PCL resulted in increasing the size of the fiber. This was in agreement with another study where the addition of nanoparticle increased the diameter of fiber [18]. However, many agglomerations were found within the random fiber (Figures 1(d) and 1(f)). Another study showed higher agglomeration at 20\% HA into different polymer of poly(D,L-lactide-co-glycolide) (PLGA) had broken fiber, but in this study no broken fiber was identified [19]. 


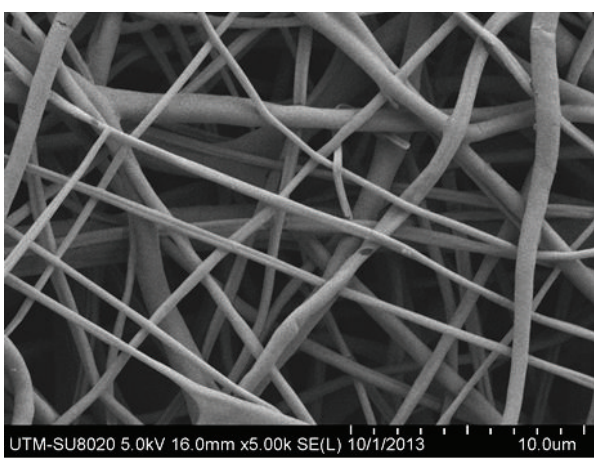

(a)

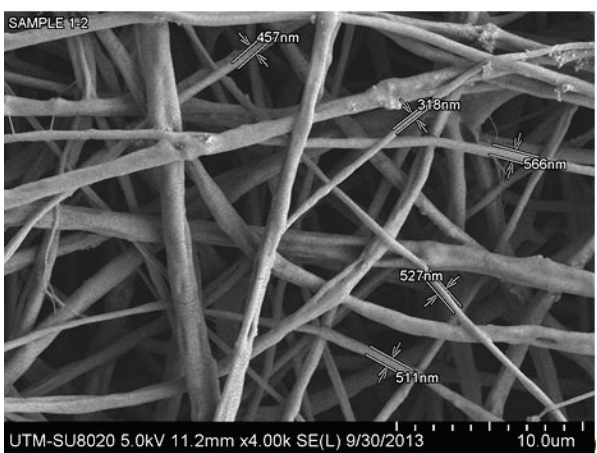

(c)

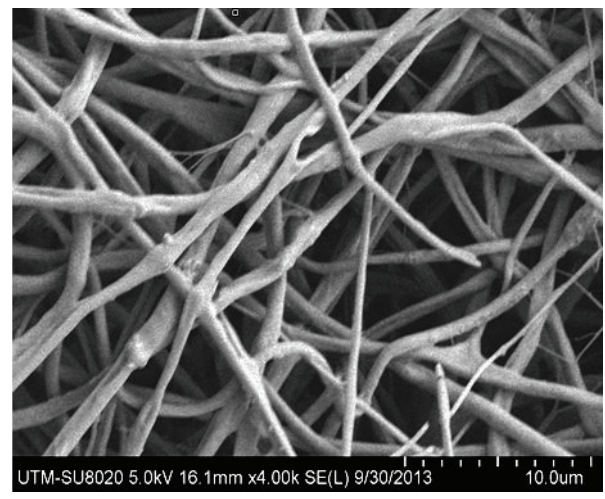

(e)

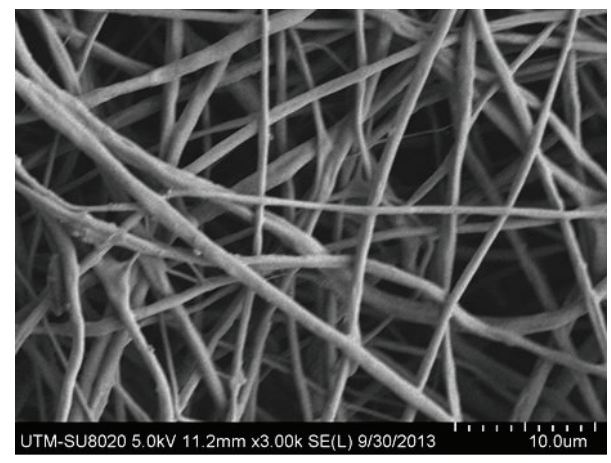

(b)

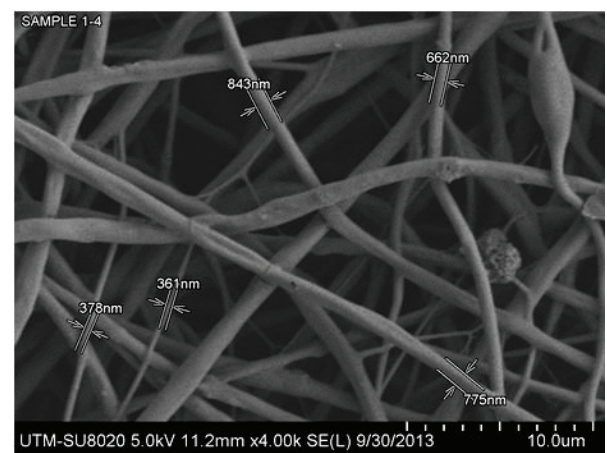

(d)

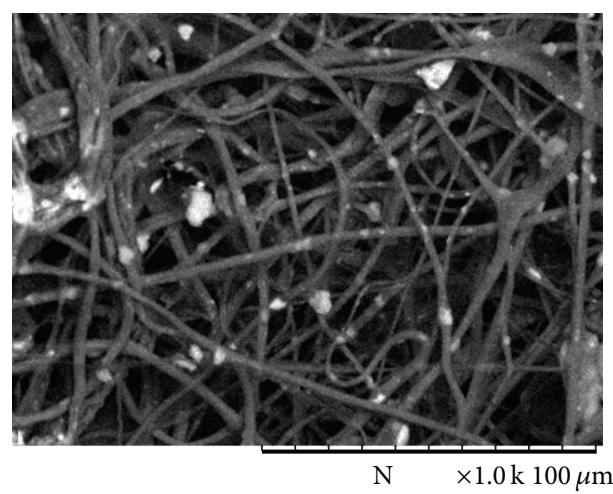

(f)

FIGURE 1: SEM micrographs of fibers at different compositions: (a) 7.5\% (w/v) PCL; (b) 12.5\% (w/v) PCL; (c) 7.5\% (w/v) PCL with 10\% (w/w) nHA; (d) 7.5\% (w/v) PCL with 20\% nHA; (e) $12.5 \%$ (w/v) PCL with 10\% (w/w) nHA; (f) $12.5 \%$ (w/v) PCL with $10 \%$ (w/w).

3.2. Bioactivity Evaluation of Fibers in SBF. The ability of forming apatite onto the fiber was evaluated through soaking them in SBF for 3, 7, and 14 days using a sample of 7.5\% (w/v) PCL and 7.5\% (w/v) PCL with 10\% (w/w) nHA. When the sample was immersed into SBF, PCL fiber was floated suggesting that high hydrophobic surface prevents water absorption. Meanwhile, composite fiber settled down to the bottom dish suggesting the fiber was high in density. It also showed faster absorption of water into the fiber. From Figure 2, it was observed that the composite fiber started to nucleate hydroxyapatite at day 3 and showed full mineralization after 7 days. After 14 days, some parts of fibers were completely covered by the apatite layer and the interfaces of fibers were blocked by apatite layer. Meanwhile, no noticeable bone-apatite layer was observed on PCL fibers even after seven days. A scaffold material should possess porous structure with high percentage of porosity and good interconnectivity. The fiber should maintain the pore shape to provide better connectivity as a scaffold. Thus, the fibers after immersing for 7 days in SBF had good property as a scaffold as it showed better interconnectivity of the pores while preserving its microstructure. EDX analyses at different locations of composite electrospun fibers also confirmed the presence of nHA particles that were well mixed with polymer fiber surfaces (Figure 3(a)). The distribution of $\mathrm{Ca}$ in $10 \% \mathrm{nHA}$ in PCL nanofiber matrices after immersing in SBF was further confirmed by the elemental mapping of $\mathrm{Ca}$ (Figure 3(b)). 


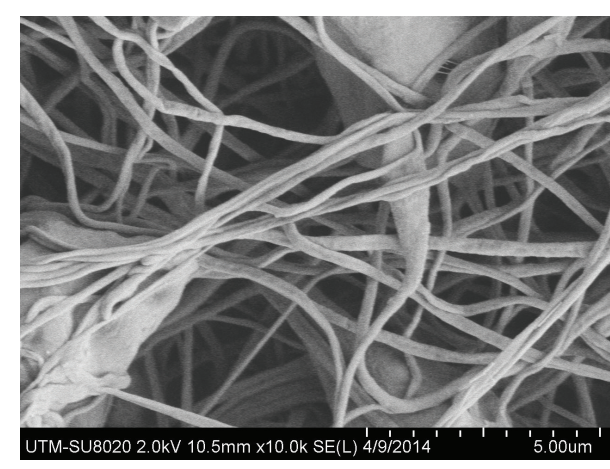

(a)

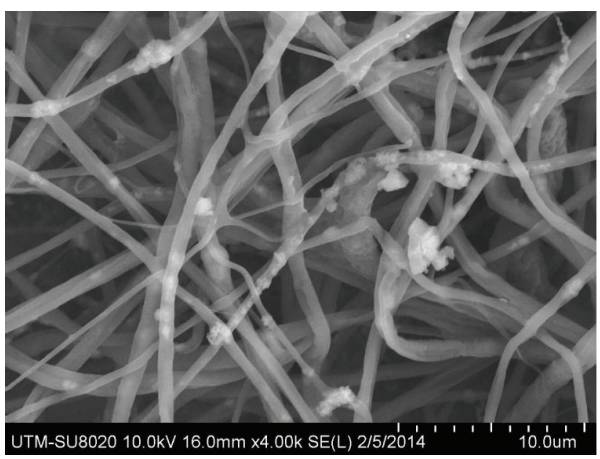

(c)

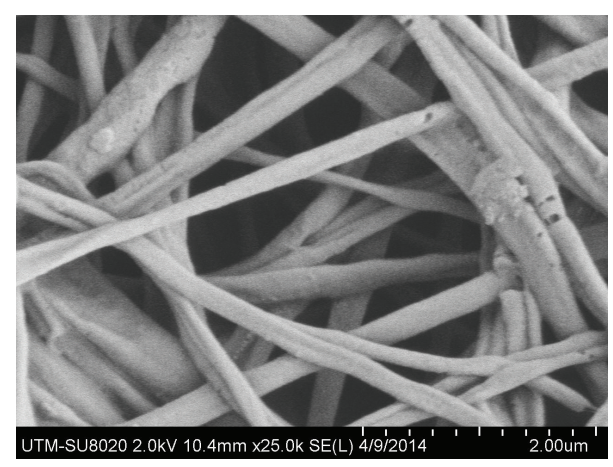

(b)

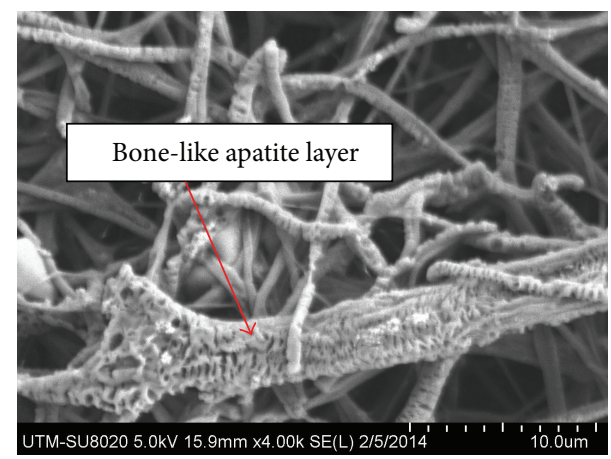

(d)

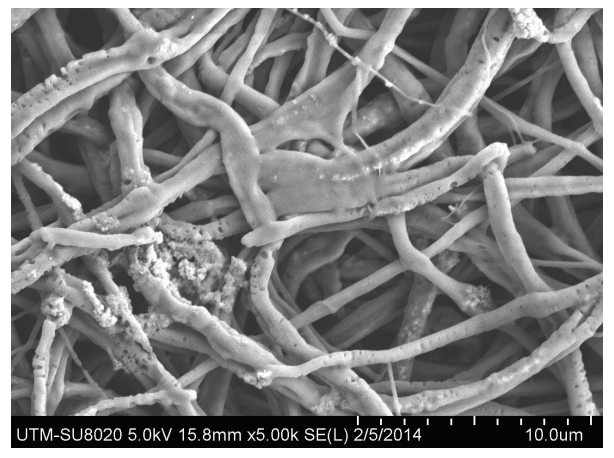

(e)

FIGURE 2: FESEM micrographs of 7.5\% (w/v) PCL fiber immersed in SBF after (a) 3 days, (b) 7 days; (c) 7.5\% (w/v) PCL with 10\% (w/w) nHA immersed in SBF after 3 days, (d) 7 days, and (e) 14 days.

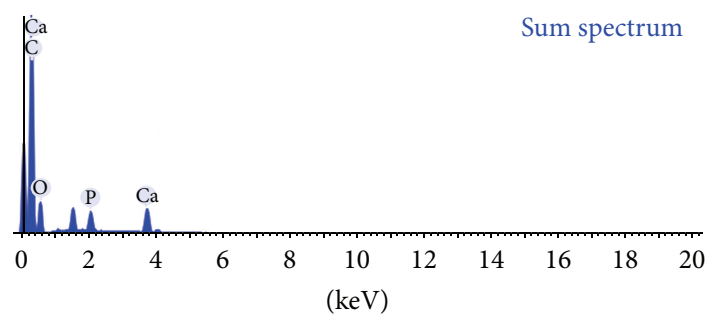

Full scale 20884 cts cursor: 0.000

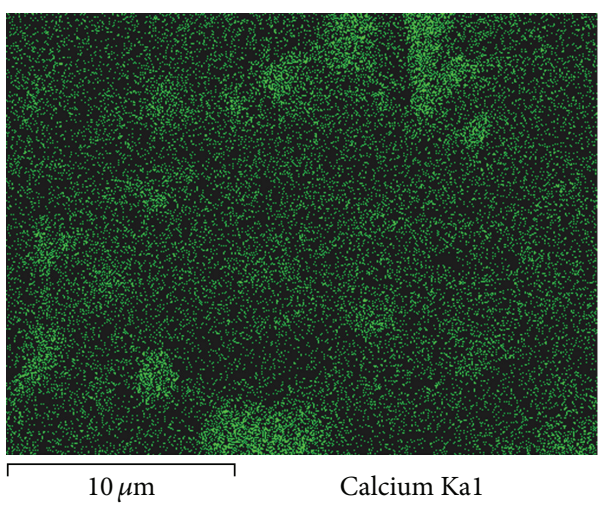

(b)

FIgURE 3: (a) An EDX spectrum and (b) Ca distribution on PCL with 10\% (w/w) nHA immersed in SBF after 7 days. 


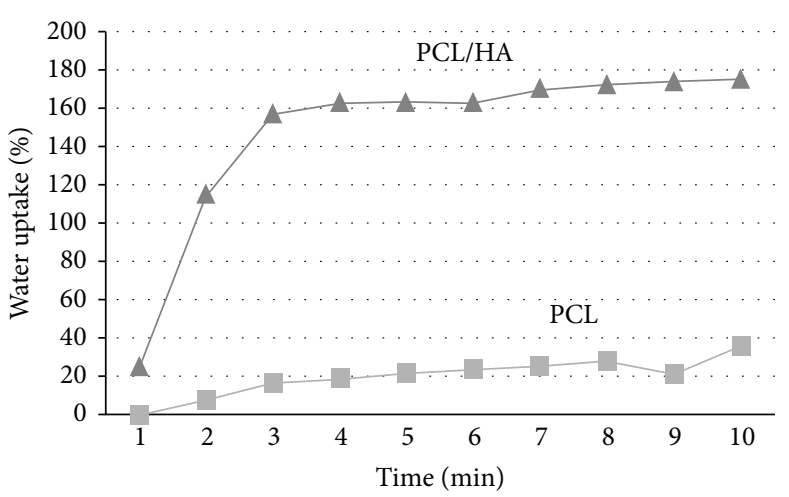

(a)

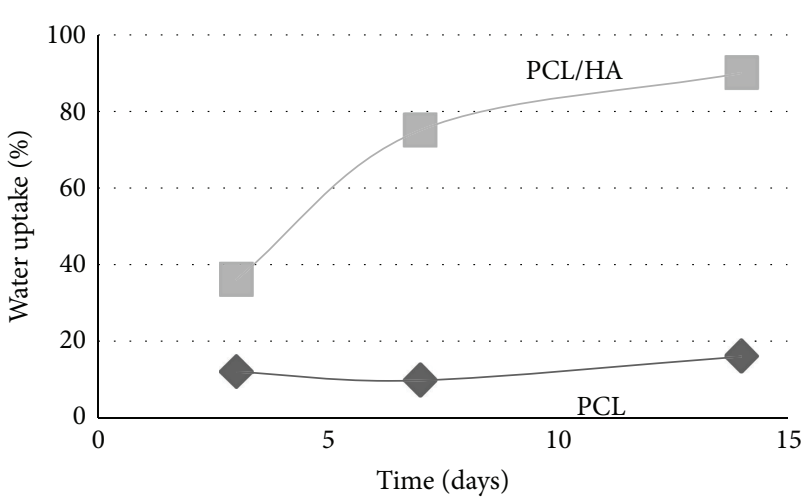

(b)

FIGURE 4: Water uptake of PCL fiber and PCL/HA fibers in (a) deionized water over time (minutes) and (b) in simulated body fluid (SBF) over time (days).

TABLE 1: Contact angle measurement of fibers at different PCL and HA concentration.

\begin{tabular}{|c|c|c|c|c|c|c|}
\hline Composition & $0 \% \mathrm{HA} / 7.5 \%$ PCL & $\begin{array}{c}10 \% \mathrm{HA} / 7.5 \% \\
\text { PCL }\end{array}$ & $\begin{array}{c}20 \% \text { HA/7.5\% } \\
\text { PCL }\end{array}$ & $\begin{array}{c}0 \% \text { HA } / 12.5 \% \\
\text { PCL }\end{array}$ & $\begin{array}{c}10 \% \text { HA } / 12.5 \% \\
\text { PCL }\end{array}$ & $\begin{array}{c}20 \% \mathrm{HA} / 12.5 \% \\
\text { PCL }\end{array}$ \\
\hline Contact angle & $127.3 \pm 2.4$ & $112.7 \pm 3.1$ & $106.3 \pm 4.5$ & $120.5 \pm 2.2$ & $115.1 \pm 4.1$ & $93.7 \pm 3.6$ \\
\hline
\end{tabular}

3.3. Contact Angle Measurement. The wettability of the fiber was determined using contact angle. The desired scaffold property for cell interaction is hydrophilic surface. Table 1 shows the contact angle of different composition of HA/PCL fibers. The change in contact angle was observed. With an increasing amount of $10 \%(\mathrm{w} / \mathrm{w})$ and $20 \%(\mathrm{w} / \mathrm{w}) \mathrm{nHA}$, some decreases of contact angles were observed. This is due to the incorporation of hydrophilic nHA. This shows that the incorporation of $\mathrm{nHA}$ as a second-phase material with hydrophilic property reduced the contact angle of pure PCL fiber. Also surface roughness had influence on this property $[19,20]$. These results indicated that PCL/nHA fibers had better hydrophilicity. A change in contact angle of the fiber can give early prediction of successful blending and surface modification. However, it also depends on the surface roughness of the fiber. This is because when the surface roughness is high, only a small quantity of water may come in contact with the surface of the fiber. So, the interaction between fiber and water becomes low and the interaction of water and air becomes high. This will cause increase in contact angle for rougher surface. It was also reported that the surface roughness increased with increasing of fiber diameter and increasing with the incorporation of nHA [21, 22]. Since PCL is hydrophobic polymer, adding nHA into the polymer can alter the properties into more hydrophilic and rougher surface. Rougher surface may cause slightly higher contact angle of fiber. However since HA is hydrophilic, there was only a slight change of lower contact angle with the increasing amount of nHA. The contact angle of PCL/nHA fiber after
7 days in SBF was 84.4 \pm 3.5. The deposition of apatite on fiber surface rendered them into more hydrophilic one.

3.4. Water Uptake. Figure 4(a) shows water uptake of $7.5 \%(\mathrm{w} / \mathrm{v})$ PCL and 7.5\% (w/v) PCL with $10 \%(\mathrm{w} / \mathrm{w}) \mathrm{nHA}$ fiber at different time interval after soaking in deionized water. It showed that PCL/nHA fiber had higher water uptake while PCL had lower water uptake properties. Initial water uptake of PCL fiber was $0.4 \%$ and had the highest water uptake at $35.5 \%$. Meanwhile, PCL/nHA fiber had initial water uptake of $25 \%$ and the highest water uptake of $175 \%$.

The water uptake of the fiber immersed in SBF was measured as in Figure 4(b). PCL fiber showed a lower percentage of water absorbed (11.94\%) at day 3 due to hydrophobic property. From day 3 to day 7, it was slightly decreased by $9.72 \%$. Up to day 14 , it was gradually increased to $15.95 \%$. PCL/nHA fiber showed faster water absorption at day 3 with $36 \%$ water uptake. From day 3 to day 7, it showed an abrupt increase by $75 \%$. The water uptake gradually increased until reaching a final value of $90 \%$ at day 14 . The results showed PCL/nHA exhibits better hydrophilicity than PCL fiber. After 20 days, no significant increase in water uptake was observed, indicating the saturation state. The water uptake properties are very important for porous materials to study the mechanism. After an initial period of time, all the materials exhibit an equilibrium state [4]. After longer time, when the polymers start to degrade or the HA starts to dissolute, weight loss can be observed for composite scaffolds or fibers. 


\section{Conclusion}

Using the electrospinning technique, beadless HA/PCL nanofiber was obtained. Immersion in SBF showed 7.5\% (w/v) PCL with $10 \%(\mathrm{w} / \mathrm{w}) \mathrm{nHA}$ fiber had good bioactivity up to seven days compared to PCL fiber. Contact angle and water uptake showed that the composite PCL/nHA fibers were more hydrophilic than PCL fiber. The water uptake property was also greater in the composite fibers than pure PCL fiber. The composite electrospun fiber of PCL with osteoconductive nHA is expected to be conducive for osteoblast growth for bone tissue regeneration.

\section{Conflict of Interests}

The authors declare that there is no conflict of interests.

\section{Acknowledgments}

The authors would like to acknowledge the Faculty of Biosciences and Medical Engineering, Universiti Teknologi Malaysia (UTM), for the lab facilities. This work was supported by research Grants GUP Tier 1 (05H07). The authors are also thankful for the support given by MOHE, RMC, and UTM.

\section{References}

[1] T. J. Webster and E. S. Ahn, "Nanostructured biomaterials for tissue engineering bone," Advances in Biochemical Engineering/Biotechnology, vol. 103, pp. 275-308, 2007.

[2] V. Leung and F. Ko, "Biomedical applications of nanofibers," Polymers for Advanced Technologies, vol. 22, no. 3, pp. 350-365, 2011.

[3] L. T. H. Nguyen, S. Chen, N. K. Elumalai et al., "Biological, chemical, and electronic applications of nanofibers," Macromolecular Materials and Engineering, vol. 298, no. 8, pp. 822867, 2013.

[4] N. Sultana and T. H. Khan, "Water absorption and diffusion characteristics of nanohydroxyapatite (nHA) and poly(hydroxybutyrate-co-hydroxyvalerate-) based composite tissue engineering scaffolds and nonporous thin films," Journal of Nanomaterials, vol. 2013, Article ID 479109, 8 pages, 2013.

[5] T. Sun, T. H. Khan, and N. Sultana, "Fabrication and in vitro evaluation of nanosized hydroxyapatite/Chitosan-based tissue engineering scaffolds," Journal of Nanomaterials, vol. 2014, Article ID 194680, 8 pages, 2014.

[6] L. A. Bosworth and S. Downes, "Acetone, a sustainable solvent for electrospinning poly ( $\varepsilon$-caprolactone) fibres: effect of varying parameters and solution concentrations on fibre diameter," Journal of Polymers and the Environment, vol. 20, no. 3, pp. 879886, 2012.

[7] A. Cipitria, A. Skelton, T. R. Dargaville, P. D. Dalton, and D. W. Hutmacher, "Design, fabrication and characterization of PCL electrospun scaffolds-a review," Journal of Materials Chemistry, vol. 21, no. 26, pp. 9419-9453, 2011.

[8] L. S. Nair and C. T. Laurencin, "Biodegradable polymers as biomaterials," Progress in Polymer Science, vol. 32, no. 8-9, pp. 762-798, 2007.
[9] C. H. Kim, M. S. Khil, H. Y. Kim, H. U. Lee, and K. Y. Jahng, "An improved hydrophilicity via electrospinning for enhanced cell attachment and proliferation," Journal of Biomedical Materials Research B: Applied Biomaterials, vol. 78, no. 2, pp. 283-290, 2005.

[10] W. Song, D. C. Markel, S. Wang, T. Shi, G. Mao, and W. Ren, "Electrospun polyvinyl alcohol-collagen-hydroxyapatite nanofibers: a biomimetic extracellular matrix for osteoblastic cells," Nanotechnology, vol. 23, no. 11, Article ID 115101, 2012.

[11] Y. Zhang, J. R. Venugopal, A. El-Turki, S. Ramakrishna, B. $\mathrm{Su}$, and C. T. Lim, "Electrospun biomimetic nanocomposite nanofibers of hydroxyapatite/chitosan for bone tissue engineering," Biomaterials, vol. 29, no. 32, pp. 4314-4322, 2008.

[12] H. Kim, H. Lee, and J. C. Knowles, "Electrospinning biomedical nanocomposite fibers of hydroxyapatite/poly(lactic acid) for bone regeneration," Journal of Biomedical Materials Research A, vol. 79, no. 3, pp. 643-649, 2006.

[13] M. I. Hassan, M. Mokhtar, N. Sultana, and T. H. Khan, "Production of hydroxyapatite(HA) nanoparticle and HA/PCL tissue engineering scaffolds for bone tissue engineering," in Proceedings of the 2nd IEEE-EMBS Conference on Biomedical Engineering and Sciences (IECBES '12), pp. 239-242, December 2012.

[14] W. Y. Zhou, M. Wang, W. L. Cheung, B. C. Guo, and D. M. Jia, "Synthesis of carbonated hydroxyapatite nanospheres through nanoemulsion," Journal of Materials Science: Materials in Medicine, vol. 19, no. 1, pp. 103-110, 2008.

[15] T. Kokubo, H. Kim, and M. Kawashita, "Novel bioactive materials with different mechanical properties," Biomaterials, vol. 24, no. 13, pp. 2161-2175, 2003.

[16] B. Rai, S. H. Teoh, and K. H. Ho, "An in vitro evaluation of PCLTCP composites as delivery systems for platelet-rich plasma," Journal of Controlled Release, vol. 107, no. 2, pp. 330-342, 2005.

[17] M. Kharaziha, M. H. Fathi, and H. Edris, "Development of novel aligned nanofibrous composite membranes for guided bone regeneration," Journal of the Mechanical Behavior of Biomedical Materials, vol. 24, pp. 9-20, 2013.

[18] P. Wutticharoenmongkol, N. Sanchavanakit, P. Pavasant, and P. Supaphol, "Preparation and characterization of novel bone scaffolds based on electrospun polycaprolactone fibers filled with nanoparticles," Macromolecular Bioscience, vol. 6, no. 1, pp. 70-77, 2006.

[19] M. V. Jose, V. Thomas, K. T. Johnson, D. R. Dean, and E. Nyairo, "Aligned PLGA/HA nanofibrous nanocomposite scaffolds for bone tissue engineering," Acta Biomaterialia, vol. 5, no. 1, pp. 305-315, 2009.

[20] F. Yang, J. G. C. Wolke, and J. A. Jansen, "Biomimetic calcium phosphate coating on electrospun poly( $\varepsilon$-caprolactone) scaffolds for bone tissue engineering," Chemical Engineering Journal, vol. 137, no. 1, pp. 154-161, 2008.

[21] C. Xu, F. Yang, S. Wang, and S. Ramakrishna, "In vitro study of human vascular endothelial cell function on materials with various surface roughness," Journal of Biomedical Materials Research A, vol. 71, no. 1, pp. 154-161, 2004.

[22] V. Thomas, D. R. Dean, M. V. Jose, B. Mathew, S. Chowdhury, and Y. K. Vohra, "Nanostructured biocomposite scaffolds based on collagen coelectrospun with nanohydroxyapatite," Biomacromolecules, vol. 8, no. 2, pp. 631-637, 2007. 

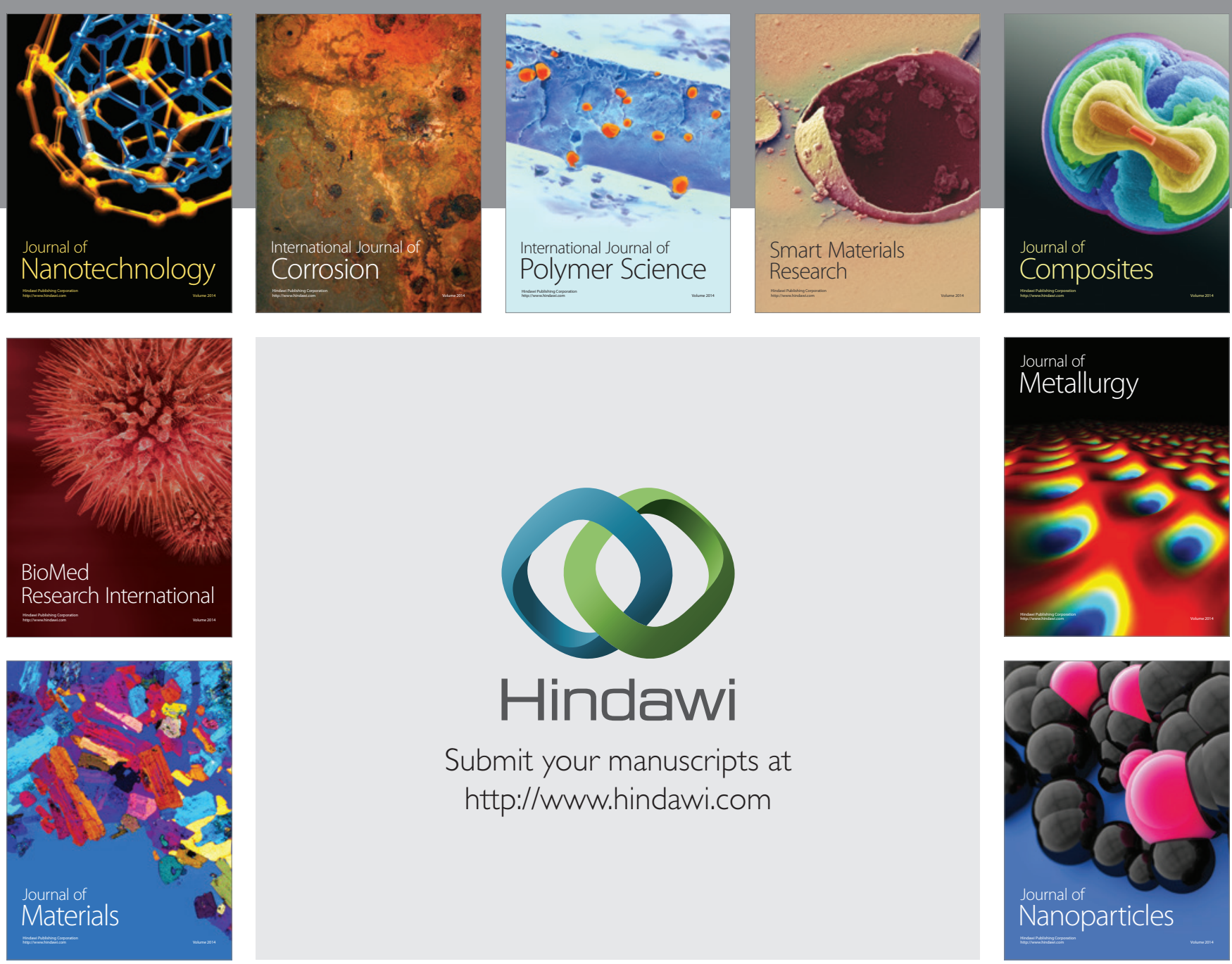

Submit your manuscripts at http://www.hindawi.com
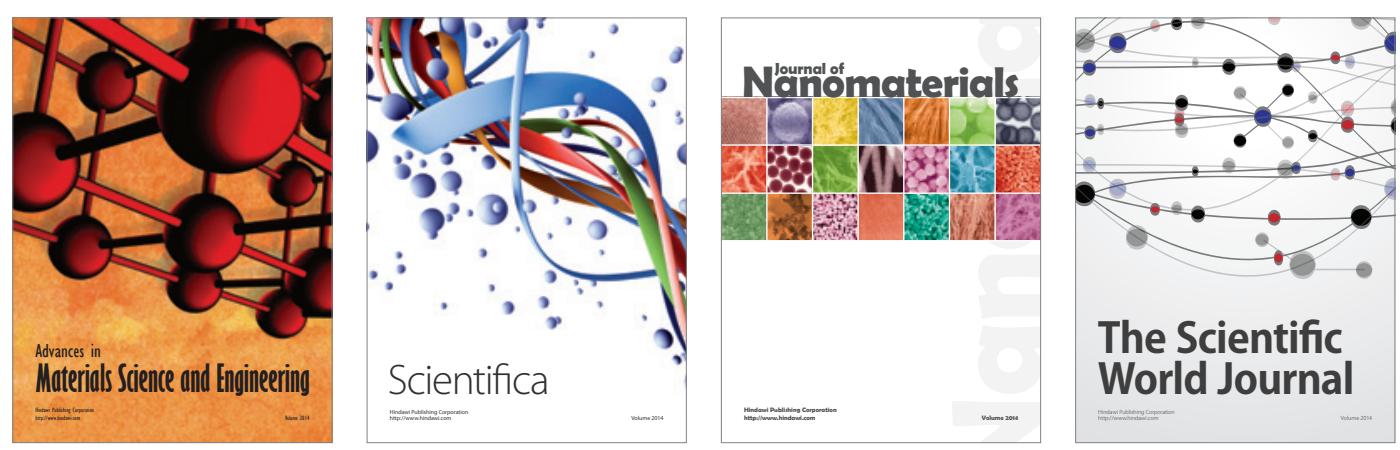

\section{The Scientific World Journal}
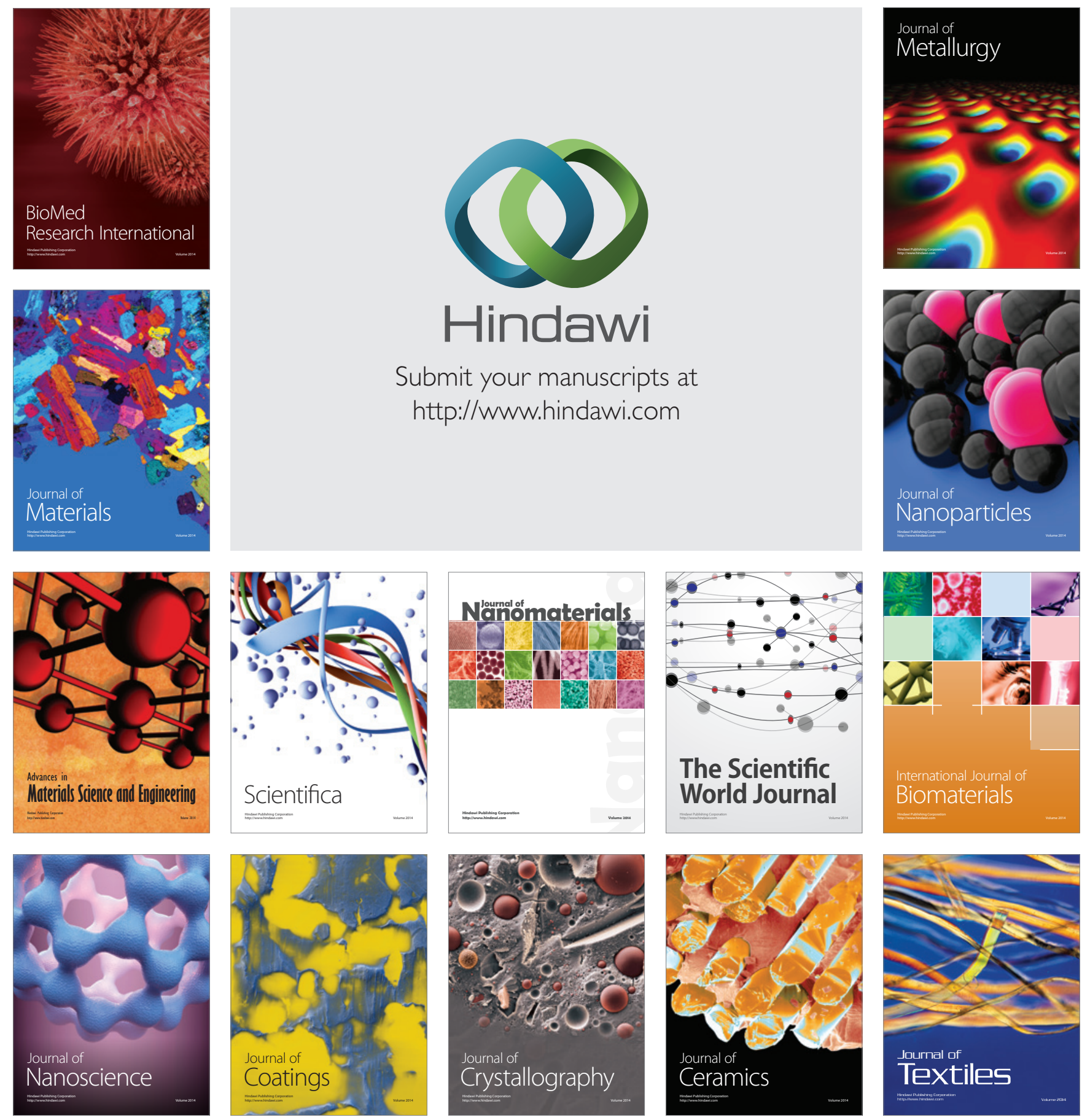